\title{
openheart Rationale for the ASSAIL-MI-trial: a randomised controlled trial designed to assess the effect of tocilizumab on myocardial salvage in patients with acute ST-elevation myocardial infarction (STEMI)
}

Anne Kristine Anstensrud (D) ,1,2 Sindre Woxholt,, ${ }^{3,4}$ Kapil Sharma, ${ }^{5}$ Kaspar Broch, ${ }^{1,6}$ Bjørn Bendz, ${ }^{1,2}$ Svend Aakhus, ${ }^{4}$ Thor Ueland, ${ }^{7,8}$ Brage H Amundsen, ${ }^{3,4}$ Jan Kristian Damås, ${ }^{9,10}$ Einar Hopp, ${ }^{11}$ Ola Kleveland, ${ }^{3,4}$ Knut Haakon Stensæth, ${ }^{4,12}$ Anders Opdahl, ${ }^{1}$ Nils-Einar Kløw, ${ }^{2,13}$ Ingebjørg Seljeflot, ${ }^{2,14}$ Geir Øystein Andersen, ${ }^{5,14}$ Rune Wiseth,, ${ }^{3,4}$ Pål Aukrust, ${ }^{2,15}$ Lars Gullestad ${ }^{1,2}$

To cite: Anstensrud AK Woxholt S, Sharma K, et al. Rationale for the ASSAIL-MItrial: a randomised controlled trial designed to assess the effect of tocilizumab on myocardial salvage in patients with acute ST-elevation myocardial infarction (STEMI). Open Heart 2019;6:e001108. doi:10.1136/ openhrt-2019-001108

Received 9 June 2019 Accepted 12 September 2019

Check for updates

(C) Author(s) (or their employer(s)) 2019. Re-use permitted under CC BY-NC. No commercial re-use. See rights and permissions. Published by BMJ.

For numbered affiliations see end of article.

Correspondence to Anne Kristine Anstensrud; annans@ous-hf.no

\section{ABSTRACT}

Introduction Interleukin-6 (IL-6) may be involved in ischaemia-reperfusion injury and myocardial remodelling after myocardial infarction (MI). We have recently shown that IL-6 inhibition by tocilizumab attenuates systemic inflammation and troponin T-release in patients with acute non-ST elevation MI (NSTEMI). Experimental studies suggest that IL-6 inhibition can limit infarct size through anti-inflammatory mechanisms, but this has not been tested in clinical studies. With the ASSessing the effect of Anti-IL-6 treatment in MI (ASSAIL-MI) trial, we aim to examine whether a single administration of the IL-6 receptor antagonist tocilizumab can increase myocardial salvage in patients with acute ST-elevation MI (STEMI).

Methods and analysis The ASSAIL-MI trial is a randomised, double blind, placebo-controlled trial, conducted at three high-volume percutaneous coronary intervention (PCl) centres in Norway. 200 patients with first-time STEMI presenting within 6 hours of the onset of chest pain will be randomised to receive tocilizumab or matching placebo prior to $\mathrm{PCl}$. The patients are followed-up for 6 months. The primary endpoint is the myocardial salvage index measured by cardiac MRI (CMR) 3-7 days after the intervention. Secondary endpoints include final infarct size measured by CMR and plasma markers of myocardial necrosis. Efficacy and safety assessments during follow-up include blood sampling, echocardiography and CMR.

Ethics and dissemination Based on previous experience the study is considered feasible and safe. If tocilizumab increases myocardial salvage, further endpoint-driven multicentre trials may be initiated. The ASSAIL-MI trial has the potential to change clinical practice in patients with STEMI.

Registration Clinicaltrials.gov, identifier NCT03004703.

\section{Key questions}

What is already known about this subject?

- Interleukin-6 (IL-6) may be involved in ischaemia-reperfusion injury and myocardial remodelling after myocardial infarction. Experimental studies suggest that IL-6 inhibition can limit infarct size through anti-inflammatory mechanisms.

What does this study add?

- The trial is designed to test the hypothesis that treatment with tocilizumab, an IL-6 receptor antagonist before and during $\mathrm{PCl}$ will increase myocardial salvage in patients presenting with acute ST-elevation myocardial infarction (STEMI).

How might this impact on clinical practice?

- If tocilizumab increases myocardial salvage, IL-6 inhibition might be an adjuvant therapeutic target for patients with STEMI. Larger endpoint-driven multicentre trials may be initiated.

\section{INTRODUCTION}

Myocardial infarction (MI) is a major cause of morbidity and mortality in the Western world. ${ }^{12}$ Infarct size is the main determinant of death and complications after MI. Limiting the extent of the myocardial necrosis has therefore been an important objective. In patients presenting with acute ST segment elevation MI (STEMI), urgent myocardial reperfusion with percutaneous coronary intervention (PCI) is the most effective treatment to this end. However, the morbidity and mortality in patients with STEMI remain substantial. ${ }^{12}$ Other adjuvant strategies are therefore required to reduce infarct size 


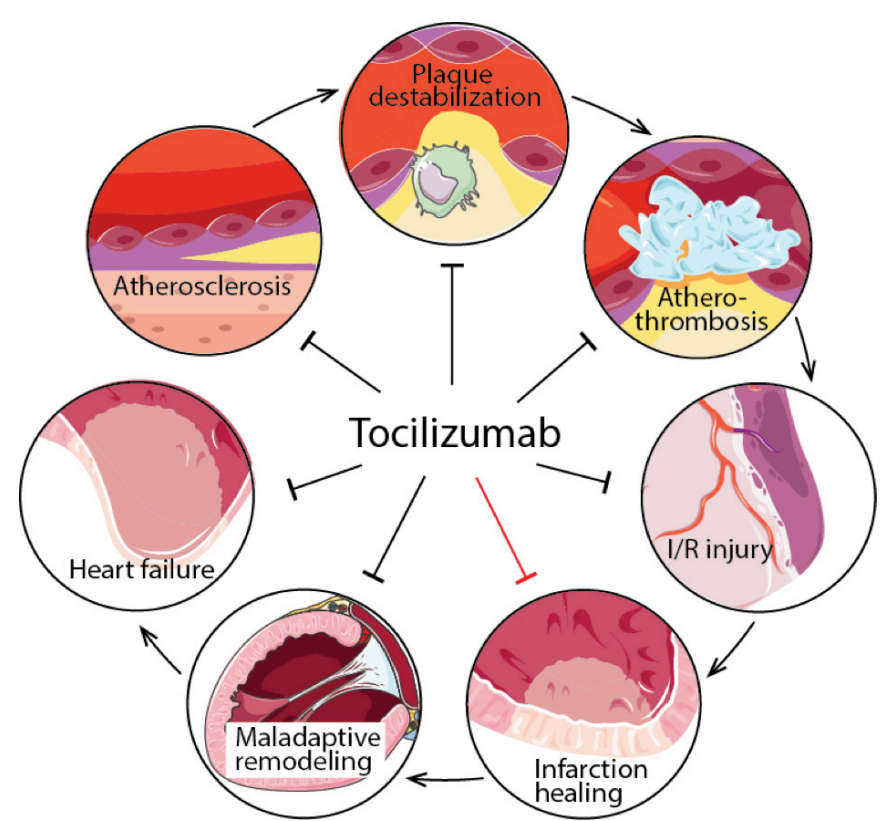

Figure 1 The figure shows possible actions for interleukin- 6 (IL-6) and targets for the anti-IL-6 antagonist, tocilizumab in the atherothrombotic process. Tocilizumab could (1) reduce initiation and progression of the atherosclerotic process, (2) stabilise atherosclerotic plaques, (3) inhibit initiation and propagation of the thrombus, (4) reduce ischaemia/ reperfusion (I/R) injury, (5) inhibit the maladaptive left ventricular remodelling process and (6) prevent development of symptomatic heart failure. However, tocilizumab could potentially also attenuate infarct healing.

and improve outcomes. The role of inflammation in plaque destabilisation and post-MI myocardial remodelling has been thoroughly investigated. Several studies have also examined the role of IL-6 in these processes. However, at present, there are no data on the effect of IL-6 inhibition in patients with STEMI.

We recently conducted a double blind, placebo controlled trial in 117 patients with non-ST segment elevation MI (NSTEMI) who presented at a median of 2 days after the onset of chest pain. In this study, a single, intravenous dose of the IL-6 receptor antagonist tocilizumab reduced C-reactive protein (CRP) levels by more than $50 \%$ during hospitalisation. Importantly, tocilizumab also reduced PCI-related troponin T (TnT) levels, suggesting that tocilizumab reduced periprocedural myocardial injury. ${ }^{3}$ The favourable effects of tocilizumab were limited to patients who underwent PCI and patients who were randomised within 2 days after symptom debut. However, as expected in patients with non-STEMI (NSTEMI), the peak TnT plasma concentrations were relatively low (approximately $300 \mathrm{ng} / \mathrm{L}$ before baseline), and the study was not designed to examine the effect on myocardial remodelling or infarct size. The beneficial effect of tocilizumab in the NSTEMI population may reflect an effect on periprocedural myocardial injury (PMI) following PCI, and a stabilising effect on the affected coronary plaque, rather than an effect on infarct size and myocardial remodelling. Thus, while our recent study indicates that IL-6 inhibition has largely favourable effects in NSTEMI, it is unknown whether similar, beneficial effects can be obtained in patients with STEMI. The pathophysiologies in these patients have similarities, but also differences. ${ }^{4}$ On this background, we wanted to investigate the effect of a single fixed dose of tocilizumab $(280 \mathrm{mg})$ in patients with acute STEMI. We postulate that a single dose of tocilizumab (RoActemra) will have favourable effects on myocardial salvage, as assessed by cardiac MRI (CMR) and markers of myocardial necrosis, without negative consequences for the myocardial repair process in these patients.

The ASSessing the effect of Anti-IL-6 treatment in Myocardial Infarction (ASSAIL-MI) trial is a randomised, controlled, double blind, parallel arm trial designed to test the hypothesis that treatment with tocilizumab before and during PCI will increase myocardial salvage in patients presenting with acute STEMI. Our ambition is to improve the prognosis of patients with STEMI, and if we fulfil this ambition, our research has the potential to change clinical practice.

\section{Acute coronary syndromes (ACS), ischaemia/reperfusion (I/R) injury and inflammation}

Whereas the abrupt restoration of blood flow by PCI undoubtedly has a net beneficial effect, it may also have detrimental consequences, including myocardial stunning, ventricular arrhythmias and microvascular dysfunction. ${ }^{5}$ The I/R injury may account for as much as $50 \%$ of the myocardial damage during MI. ${ }^{6}$ While the myocardial response to the reperfusion injury aims to restore homeostasis within the heart through repair and adaptive remodelling, a dysregulated process can induce maladaptive remodelling and subsequent myocardial failure. An ideal therapeutic approach to MI should (1) counteract plaque destabilisation and thrombus formation, (2) impair the I/R injury, (3) minimise infarct size and (4) inhibit maladaptive remodelling without attenuating the repair process. At present, we lack the necessary tools to fully achieve these goals.

Inflammation is involved in several aspects of coronary artery disease $(\mathrm{CAD})$ and ACS. Plaque destabilisation (ie, endothelial erosions and fibrous cap rupture) with subsequent thrombus formation and vascular obstruction is the principal cause of acute coronary events. Inflammation is involved in all these features of ACS. ${ }^{78}$ In addition, different cytokines as well as reactive oxygen species (ROS), intracellular calcium overload and myocardial infiltration of leucocytes may contribute to the reperfusion injury occurring after the opening of occluded coronary arteries. Enhanced oxidative stress activates inflammatory pathways within the myocardium, which again enhances ROS production, in a vicious cycle after PCI. ${ }^{6}$ Myocardial inflammation is also a major contributor to infarct size through the activation and release of matrix degrading enzymes and induction of cardiomyocyte and fibroblast apoptosis and necrosis. However, while the injurious role of inflammation in MI is well established, how to modulate the activation of the inflammatory responses remains unclear. While massive or sustained 


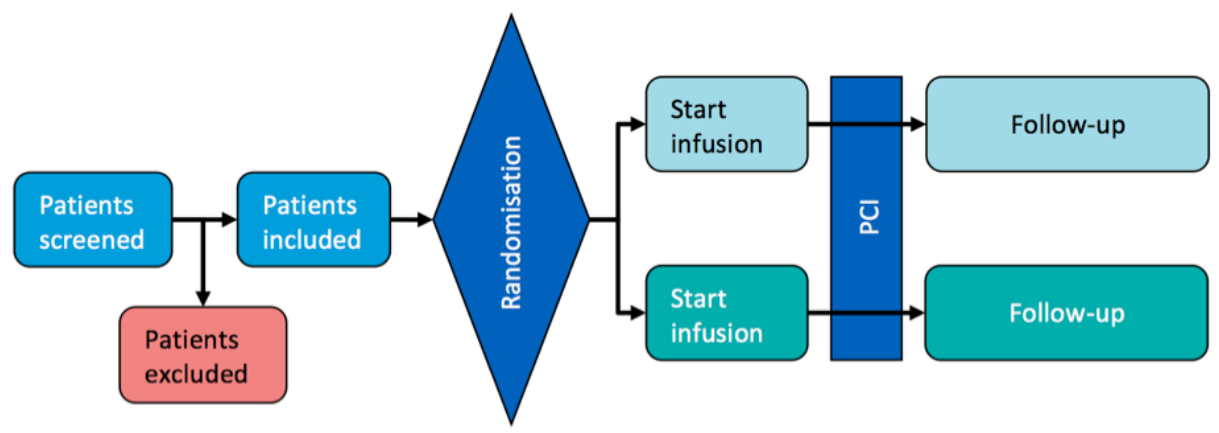

Figure 2 Overall study design.

inflammatory responses are harmful and may contribute to myocardial damage and increased infarct size, inflammation is also prerequisite for tissue repair. This dual role of inflammation following MI represents a therapeutic challenge.

\section{Therapeutic intervention during $\mathrm{MI}$}

Recently, a great breakthrough on therapeutic approaches targeting inflammation in atherosclerotic disorders was achieved with the Canakinumab Anti-inflammatory Thrombosis Outcomes Study trial. ${ }^{9}$ In this trial, anti-inflammatory therapy with the interleukin-1 beta (IL-1 $\beta$ ) antibody canakinumab led to a significantly lower rate of recurrent cardiovascular events in patients with previous MI. However, few studies have explored anti-inflammatory strategies in MI. A randomised trial evaluating IL-1 receptor (IL-1R) antagonism in NSTEMI showed a significantly lower level of CRP in the intervention arm, but not during the first 2 days after drug administration, and without effects on TnT. ${ }^{10}$ A larger multicentre study on the effect of the IL-1R antagonist anakinra in patients with STEMI is ongoing. ${ }^{11}$ In patients with STEMI, pexelizumab ( $\mathrm{n}=960)$, which inhibits the C5 complement protein $^{12}$ mitigates inflammation. Moreover, whereas pexelizumab had no measurable effect on infarct size, it showed a significant reduction in mortality. Subsequent studies, however, have failed to demonstrate effects on clinical outcomes. ${ }^{13}$ In fact, used adjunctively with fibrinolytic agents, pexelizumab did not reduce infarct size, as assessed by creatine kinase-myocardial band (MB), or the risk of adverse clinical events. ${ }^{14}$ Recently, Defteros and colleagues showed that in patients with STEMI $(n=151)$, the antirheumatic drug colchicine could reduce creatine kinase-MB, and, in a subgroup of patients, infarct size as assessed by CMR. ${ }^{15}$ Moreover, inclacumab targeting P-selectin, an adhesion molecule that facilitates the interaction between platelets, endothelial cells and leucocytes, was found to reduce TnI after PCI in patients with NSTEMI $(n=322) .{ }^{16}$ More recently, the p38 mitogen-activated protein kinase inhibitor losmapimod, known to attenuate a range of inflammatory pathways, failed to improve outcomes in a relatively large randomised trial in patients with NSTEMI $(\mathrm{n}=3503) .{ }^{17}$

To date, there are no studies on IL-6 inhibition in patients with STEMI. Several drugs (eg, glycoprotein IIb/
IIIa antagonists) attenuate the levels of circulating IL-6 after ACS and PCI. ${ }^{18}$ However, there are studies suggesting that IL-6 is insufficiently suppressed with contemporary therapies. In the Myocardial Ischemia Reduction with Acute Cholesterol Lowering study, treatment with atorvastatin reduced the level of circulating CRP, but failed to reduce IL-6 levels. ${ }^{19}$ Moreover, numerous studies have shown that, despite 'optimal' therapy, IL-6 levels are higher in patients with ACS than in patients with stable coronary disease and healthy control subjects. In summary, there seems to be an unfulfilled need for treatment targeting IL-6. This unfulfilled need forms the scientific background for the ASSAIL-MI trial.

\section{The role of IL-6}

IL-6 is a multifunctional cytokine that is produced by a spectrum of cell types in the cardiovascular and immune systems and can influence the cardiovascular system in several ways (figure 1). It has been linked to vascular inflammation and myocardial remodelling. IL-6 and its signalling through the IL-6-receptor complex contribute to atherosclerotic plaque development and plaque destabilisation ${ }^{20}$ and has also been related to the progression of myocardial failure. ${ }^{21}$ IL-6 is a pleiotropic cytokine with a wide range of effect on immune cells and other cells with relevance for cardiovascular disease..$^{22}$ IL-6 is the main promoter of acute-phase proteins, such as CRP, and the subsequent complement activation. It causes the release of prothrombotic mediators and activation of matrix metalloproteinases. This result in matrix degradation and weakening of the atherosclerotic fibrous cap and may also have harmful effects on the repair process after MI.

IL-6 plays a significant role in myocardial remodelling predisposing to the development of overt heart failure. Experimental mice models have shown that enhanced IL-6 activation promote myocardial hypertrophy. However, deficiency of its receptor induced severe cardiac dilation..$^{23}$ These findings illustrate the balance between beneficial and harmful effects of this cytokine where too much and too little can both be harmful. Interestingly, in an experimental mice model, Kobara et al found that therapy targeting the IL-6 receptor downregulated inflammation and attenuated LV remodelling after $\mathrm{MI},{ }^{24}$ illustrating the potential for similar interventions 


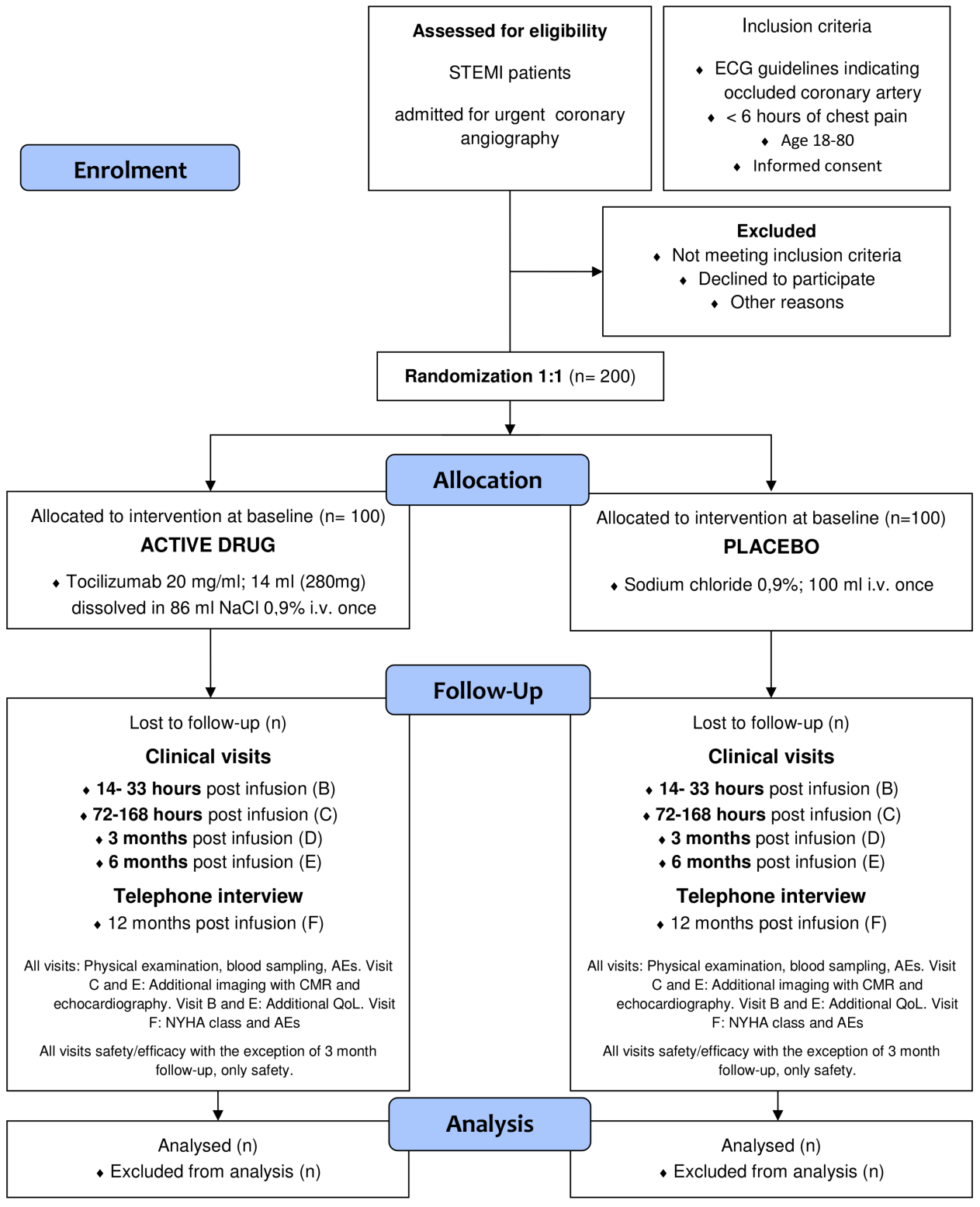

Figure 3 Study flow chart.

in humans. We have shown that high levels of soluble IL-6 receptors (ie, IL-6R and gp130) in the acute phase of STEMI are associated with subsequent major cardiac adverse events, underscoring the potential importance of the IL- 6 signalling system in patients with MI. ${ }^{25}$ To conclude, IL-6-related pathways seem to be involved in several pathogenic processes during $\mathrm{MI}$, from plaque destabilisation and thrombus formation to I/R injury and maladaptive myocardial remodelling and our hypothesis is that IL-6 inhibition has several beneficial effects in STEMI (figure 1).

\section{Known and potential benefits and risks of targeting IL-6 therapy in STEMI}

IL-6 inhibition with a single dose of tocilizumab may have several merits. First, in contrast to long-term anti-inflammatory therapy in various autoimmune disorders, we hypothesise that one dose of tocilizumab administered on admission may be sufficient to induce beneficial effects on infarct size and myocardial remodelling. Accordingly, the patients will not be exposed to long-term immunosuppression with its potential harmful effects. Second, in contrast to IL-1 $\beta$, which is an upstream inflammatory cytokine, IL-6 is a more downstream mediator. Although the effect of IL-1 $\beta$ inhibition seems at least partly to be mediated through reduction of levels of IL- $6,{ }^{26}$ we found only minor alterations in the cytokine network during tocilizumab intervention in patients with NSTEMI, suggesting a more narrow effect of IL-6 inhibition. ${ }^{27}$ We therefore expect that the targeted IL-6 therapy provided in the ASSAIL-MI trial will to a lesser degree interfere with other inflammatory pathways, some of which may be of importance for infarct healing. Third, to balance the beneficial and harmful effect of anti-inflammatory therapy, we have chosen a moderate 
and fixed dose of tocilizumab $(280 \mathrm{mg})$ that will provide complete IL-6 blockade of approximately 2 weeks. Notably, in the NSTEMI study, we found no association between body mass index and the effect of tocilizumab on CRP, suggesting that its effect is influenced by body weight to a minor degree only. ${ }^{3}$ There are several potential challenges with anti-IL6 inhibition during STEMI. We know that tocilizumab is associated with an increased risk of infections. However, this risk is probably low after a single dose only. In the NSTEMI study, we recorded no severe infectious complications in patients receiving tocilizumab. ${ }^{3}$ Tocilizumab induced a significant reduction in blood levels of neutrophils, ${ }^{3}$ but based on the role of these cells during MI, this could be a beneficial rather than a harmful effect. ${ }^{28}$ As discussed above, there is also increased risk of impaired healing after MI during IL-6 inhibition, potentially increasing the risk of myocardial rupture and cardiac dilation. However, we consider this risk to be small in patients treated within 6 hours of symptom onset. Finally, there have been some concern about the hyperlipidaemic effects of tocilizumab, but hyperlipidaemia was not observed in the NSTEMI trial. This may reflect differences in the interaction between inflammation and lipid metabolism between patients with $\mathrm{CAD}$ and patients with autoimmune disorders like rheumatoid arthritis, potentially related to the fact that nearly all patients with CAD receive statins. ${ }^{29}$

\section{METHODS AND ANALYSIS \\ Design}

The ASSAIL-MI trial is a phase II, double blind, randomised, placebo-controlled trial conducted at three experienced, high volume PCI centres in Norway. Participants are randomised in a 1:1 fashion to receive a single intravenous fixed dose of tocilizumab $(280 \mathrm{mg})$ tocilizumab or matching placebo prior to PCI. The study is designed to show superiority with regard to the primary endpoint in patients assigned to active treatment vs patients allocated to the placebo arm. The overall study design is presented in figure 2 and study procedures in figure 3.

\section{Eligibility}

The inclusion and exclusion criteria are presented in box 1 .

Patients with first time STEMI scheduled for acute PCI within 6 hours from the onset of chest pain are screened for eligibility on admittance at either participating site. Informed consent is obtained for all enrolled patients aged between 18 and 80, fulfilling ECG criteria for STEMI, and accepted for urgent coronary angiography with the intention to reperfuse a presumed occluded vessel within the given time limit. Haemodynamically unstable patients and patients with previous MI or severe comorbidities are not included in this trial. Exclusion criteria include concomitant diseases that we assume affect inflammation.
Box 1 The main inclusion and exclusion criteria

Inclusion criteria

- New ST elevation at the J-point in two contiguous leads (cut-points: $0.2 \mathrm{mV}$ in men and $>0.15 \mathrm{mV}$ in women in leads V2-V3 and/or $>0.1 \mathrm{mV}$ in other leads) in combination with symptoms consistent with acute myocardial infarction (MI).

- Presentation within 6 hours of chest pain.

- Indication for urgent coronary angiography with intent to reperfuse presumed occluded vessel.

- Age between 18 and 80 years.

- Informed consent obtained and documented according to International Council for Harmonisation of Technical Requirements for Registration of Pharmaceuticals for Human Use (ICH)/Good Clinical Practice and national/local regulations.

\section{Exclusion criteria}

- Non-ST elevation MI (NSTEMI) (non-ST segment elevation in ECG).

- Left bundle branch block in ECG.

- History of previous MI.

- Cardiogenic shock.

- Fibrinolytic therapy within 72 hours prior to admission.

- Cardiac arrest/ventricular fibrillation.

- History of severe renal failure with estimated glomerular filtration rate $<30 \mathrm{~mL} / \mathrm{min}$.

- Known, current liver disease.

- History of concurrent inflammatory, biliary obstructive or malignant disease.

- Ongoing infection.

- A history of chronic or concurrent infectious disease, including a history of HIV, tuberculosis or hepatitis B or C.

- Known, uncontrolled lower gastrointestinal (Gl) disease such as diverticulitis, Crohn's disease, ulcerative colitis or other symptomatic lower Gl conditions that could predispose to Gl perforations.

- Major surgery within 8 weeks prior to or after baseline.

- History of central nervous system demyelinating or seizure disorders.

- History of primary or secondary immunodeficiency.

- Treatment with immunosuppressants other than low dose corticosteroids (equivalent to $5 \mathrm{mg}$ of prednisone or less) at the time of randomisation.

- Immunisation with a live/attenuated vaccine within 4 weeks prior to baseline.

- History of severe allergic or anaphylactic reactions to humanised or murine monoclonal antibodies or to tocilizumab.

- Pregnancy, possible pregnancy or breast feeding - women of childbearing potential or breastfeeding mothers cannot participate. A woman is considered of childbearing potential following menarche and until becoming postmenopausal unless permanently sterile. Permanent sterilisation methods include hysterectomy, bilateral salpingectomy and bilateral oophorectomy. A postmenopausal state is defined as no menses for 12 months without an alternative medical cause.

- Contraindications to cardiac MRI (pacemaker, cardiac resynchronisation therapy, implantable cardioverter defibrillator, certain ferromagnetic implants, severe claustrophobia, allergy to contrast medium).

- Any condition/circumstances believed to interfere with the ability to comply with protocol.

- Any reason why, in the opinion of the investigator, the patient should not participate. 


\section{Box 1 Continued}

- Failure to obtain written, informed consent by patient or next of kin, for instance in case of patient death after consent has been provided in oral.

\section{Objectives}

\section{Primary objective}

The primary objective of the ASSAIL-MI trial is to examine the effect of tocilizumab on myocardial salvage index as measured by CMR with gadolinium late enhancement 3-7 days after study drug infusion.

\section{Secondary objectives}

Secondary objectives are to assess the impact of treatment on: (1) troponin release, (2) final infarct size, (3) other biomarkers of myocardial damage (ie, aspartate aminotransferase and creatine kinase-MB), (4) CRP levels during hospitalisation, (5) markers of left ventricular (LV) remodelling and (6) safety and tolerability.

\section{Explorative endpoints}

Endpoints that could explain the mechanisms of action of tocilizumab in STEMI: (1) additional markers of inflammation, (2) markers of extracellular matrix remodelling, (3) lipid parameters, (4) markers of platelet activation and additional prothrombotic and antithrombotic parameters. Endpoints are detailed in box 2 .

\section{Rationale for study endpoint}

Infarct size is a major determinant of prognosis after MI and can be accurately measured by gadolinium late enhancement CMR.$^{30} \mathrm{CMR}$ is also the method of choice for the evaluation of LV function in this setting. Infarct size is mainly determined by two factors: the size of the myocardial territory supplied by the occluded vessel and the efficacy of the therapeutic actions taken to restore normal blood flow and minimise myocardial damage. In the present study, we will study the effect of tocilizumab on myocardial damage during reperfusion. This effect can be evaluated by the myocardial salvage index, which is calculated by indexing the area of the salvaged myocardium to the area at risk of irreversible injury, thus relating the size of the damage to the area at risk. The myocardial salvage index has recently been used in several large clinical cardioprotection trials (CHILL-MI, ${ }^{31}$ MITOCARE, ${ }^{32}$ METOCARD-CNIC, ${ }^{33}$ LIPSIA CONDITIONING).$^{34}$ The area at risk can be quantified with early gadolinium enhancement images, applying a standard CMR sequence.

In addition to infarct size and the salvage index, CMR can assess microvascular obstruction, which is considered a marker of severe myocardial damage and poor prognosis. ${ }^{35}$ Soluble markers of myocardial damage, in particular TnT which is the most established marker for assessment of myocardial damage during STEMI, will be an important secondary endpoint. Explorative endpoints will elucidate possible mechanisms of action of tocilizumab in STEMI.
Box 2 Prespecified endpoints in the ASSessing the effect of Anti-IL-6 treatment in Myocardial /nfarction trial

Primary endpoint

- The between-group difference in myocardial salvage index as measured in the acute phase by cardiac MRI (CMR) with late gadolinium enhancement (LGE).

\section{Secondary endpoints}

- The between-group differences in the area under the curve (AUC) for troponin T during index hospitalisation (baseline adjusted).

- Final infarct size as measured by CMR 6 months after randomisation.

- The between-group difference in the AUC for aspartate aminotransferase and creatine kinase-myocardial band during index hospitalisation (baseline adjusted).

- The between-group differences in the AUC of C-reactive protein during index hospitalisation (baseline adjusted).

- The extent of microvascular obstruction as measured by CMR after 3-7 days.

- Left ventricular size as assessed by CMR six6months after randomisation.

- Markers of myocardial remodelling as assessed by echocardiography.

- Baseline-adjusted N-terminal prohormone of brain natriuretic peptide at 6 months.

- Safety and tolerability as assessed by clinical assessment, blood samples and imaging.

Explorative endpoints

- Additional inflammatory and anti-inflammatory mediators.

- Markers of extracellular matrix remodelling (eg, matrix metalloproteinases and their endogenous inhibitors).

- Lipid parameters (eg, low-density lipoprotein and high-density lipoprotein cholesterol and triglycerides).

- Markers of platelet activation (eg, platelet-derived inflammatory markers) and additional prothrombotic and antithrombotic parameters (eg, tissue factor and plasminogen activator inhibitor).

\section{Statistical considerations}

This trial is designed to assess the effect of treatment with tocilizumab on myocardial salvage in patients with STEMI. In patients with first time, acute anterior wall MI treated with PCI within 12 hours of the onset of symptoms, final infarct size is typically $20 \%$ of total myocardial volume. ${ }^{36}$ Studies have shown that an absolute reduction in infarct size of approximately $3 \%-5 \%$ of myocardial volume, that is, a relative reduction of $14 \%-25 \%$, given an infarct size of $20 \%$, is associated with improved survival and a reduction in clinical events. ${ }^{37}$ We therefore consider a decrease in (1-the myocardial salvage index) of $20 \%$ (equalling $20 \%$ reduction in infarct size) to be clinically relevant. With a two-sided $\alpha$ of 0.05 and a power of $90 \%$, detection of a $20 \%$ between-group difference in (1-myocardial salvage index) requires 86 patients in each arm. ${ }^{38}$

In a recent trial on the effect of IL- 6 inhibition in patients with NSTEMI, we showed that treatment with tocilizumab was associated with reduced PCI-released release of TnT. ${ }^{3}$ In the ASSAIL-MI trial, the baseline-adjusted between-group difference in AUC for TnT will be an important secondary endpoint. In order to reduce the AUC for TnT by $25 \%$, with an $\alpha$ of 0.05 and a power of 
Table 1 Baseline characteristic of the first 100 patients in the ASSessing the effect of Anti-IL-6 study

\begin{tabular}{|c|c|}
\hline Demography & \\
\hline Age, years (range) & $61.0 \pm 8.7(38-79)$ \\
\hline Male gender, n (\%) & $80(80.0)$ \\
\hline \multicolumn{2}{|l|}{ Race/ethnicity, n (\%) } \\
\hline White/Black/Asian & $96(96.0) / 0(0.0) / 4(4.0)$ \\
\hline \multicolumn{2}{|l|}{ Relationship status, n (\%) } \\
\hline Married/single/widow(er) & $73(73.0) / 22(22.0) / 5(5.0)$ \\
\hline \multicolumn{2}{|l|}{ Work status, n (\%) } \\
\hline Working/sick leave/pensioner & $61(61.0) / 4(4.0) / 34(34.0)$ \\
\hline \multicolumn{2}{|l|}{ Cardiovascular risk factors, $\mathrm{n}(\%)$} \\
\hline Current smoker/former smoker & $40(40.0) / 26(26.0) / 34(34.0)$ \\
\hline Hypertension & $33(33.0)$ \\
\hline Diabetes mellitus & $7(7.0)$ \\
\hline Peripheral vascular disease & $2(2.0)$ \\
\hline $\begin{array}{l}\text { Chest pain to hospital arrival time, hours } \\
\text { (range) }\end{array}$ & $2.4 \pm 1.2(0.42-6.0)$ \\
\hline \multicolumn{2}{|l|}{ Concomitant medication, $\mathrm{n}(\%)$} \\
\hline $\begin{array}{l}\text { ACE-inhibitor/angiotensin receptor } \\
\text { blocker }\end{array}$ & $26(26)$ \\
\hline Aldosterine antagonist & $1(1)$ \\
\hline Anticoagulants & $4(4)$ \\
\hline Antiplatelets & $10(10)$ \\
\hline Beta blocker & $7(7)$ \\
\hline Calcium antagonist & $14(14)$ \\
\hline Diuretics & $10(10)$ \\
\hline Statin & $10(10)$ \\
\hline Other & $25(25)$ \\
\hline \multicolumn{2}{|l|}{ Clinical characteristics } \\
\hline \multicolumn{2}{|l|}{ Blood pressure, mm Hg (range) } \\
\hline Systolic & $132.3 \pm 21.5(80.0-180.0)$ \\
\hline Diastolic & $82.7 \pm 16.2(50.0-121.0)$ \\
\hline Heart rate, beats per minute (range) & $70.8 \pm 16.1(40.0-125.0)$ \\
\hline Body mass index, $\mathrm{kg} / \mathrm{m}^{2}$ (range) & $27.0(15.7-39.2) \pm 4.3$ \\
\hline \multicolumn{2}{|l|}{ Killip classification, n (\%) } \\
\hline I/II/III/IV & $95(95) / 4(4) / 0(0) / 0(0)$ \\
\hline \multicolumn{2}{|c|}{ New York Heart Association functional status, $\mathrm{n}(\%)$} \\
\hline I/II/III/IV & $62(62) / 27(27) / 3(3) / 7(7)$ \\
\hline
\end{tabular}

Data are given as number (percent) or mean \pm SD (range)

$80 \%$, we need approximately 75 patients in each arm. ${ }^{38}$ To allow for dropout and to improve the chances of obtaining significance for secondary endpoints, we therefore aim to include all together 200 patients.

All statistical tests will be performed using a two-sided $5 \%$ level of significance. Continuous efficacy variables will be analysed using independent t-tests for comparisons between the treatment arms and baseline-adjusted analysis of covariance (ANCOVA) for difference in changes. If necessary, values will be log-transformed to meet the assumptions of the tests. All analyses will be analysed according to the intention-to-treat principle. Betweengroup differences in ordinal categorical variables, such as New York Heart Association class, will be analysed using ordinal logistic regression, whereas the count variables will be assessed by Poisson regression. Demographic, efficacy and safety data will be summarised by treatment group using means, minimums, medians, maximums, IQRs and SD for continuous variables and frequency counts and percentages for categorical variables. Per-protocol analyses will be performed using the same methods as for the intention-to-treat analyses.

The between-group difference in the myocardial salvage index as measured by CMR 3-7 days after study drug infusion will be assessed by an independent Student's t-test according to the intention-to-treat principle, the statistical null hypothesis being that the myocardial salvage index does not differ between the two treatment arms. Secondary analyses will be made according to the per-protocol principle. For TnT, the baseline-adjusted between-group difference in AUC for TnT will be calculated by ANCOVA, if necessarily after log transformation. The statistical null hypothesis is that the TnT release does not differ between patients allocated to tocilizumab and patients allocated to placebo.

Safety analyses will include tabulation of the type and frequency of adverse events. Any serious adverse events will be reported with comprehensive narratives. Any value of safety laboratory parameters outside normal ranges will be identified.

\section{Study management}

A trial steering committee oversees the progress of the trial. An independent data monitoring and safety committee is responsible for the regular monitoring of trial data, and it will give advice on whether the accumulated safety data, together with the results from other relevant research, necessitate premature termination of the trial.

\section{ETHICS AND DISSEMINATION}

The ASSAIL-MI trial is designed to assess the effect of a therapeutic intervention with the aim to improve outcome in a population with considerable known mortality and morbidity. The trial is conducted according to Good Clinical Practice guidelines. Based on previous trials and drug pharmacodynamics, we do not expect a substantial number of drug-related severe adverse events. The trial complies with the Declaration of Helsinki. All patients provide written informed consent before enrolment and randomisation.

This study was initiated in February 2017 and is currently recruiting patients at all three participating sites. As of April 2019, 135 patients have been enrolled. Baseline characteristics of the first 100 of these patients are shown in table 1. 
We expect patient recruitment to be complete by the end of 2019. We expect the trial to be completed and results available in 2020.

If positive, this study may be followed by larger multicentre studies with clinical endpoints like mortality and hospitalisation as primary endpoints. The study has the potential to change clinical practice in the management of patients with ACS.

\section{Author affiliations}

${ }^{1}$ Department of Cardiology, Oslo University Hospital Rikshospitalet, Oslo, Norway ${ }^{2}$ Faculty of Medicine, Institute of Clinical Medicine, University of Oslo, Oslo, Norway ${ }^{3}$ Clinic of Cardiology, St. Olavs Hospital, Trondheim University Hospital, Trondheim, Norway

${ }^{4}$ Department of Circulation and Medical Imaging, Norwegian University of Science and Technology (NTNU), Trondheim, Norway

${ }^{5}$ Department of Cardiology, Oslo University Hospital Ullevaal, Oslo, Norway

${ }^{6}$ K.G. Jebsen Cardiac Research Centre and Centre for Heart Failure Research, University of Oslo, Oslo, Norway

${ }^{7}$ Research Institute of Internal Medicine, Oslo University Hospital Rikshospitalet, Oslo, Norway

${ }^{8} \mathrm{~K}$.G.Jebsen Thrombosis Research and Expertise Center (TREC), The Arctic University of Norway, Troms $\emptyset$, Norway

${ }^{9}$ Department of Infectious Disease, St. Olavs Hospital, Trondheim University Hospital, Oslo, Norway

${ }^{10}$ Department of Clinical and Molecular Medicine, Centre of Molecular Inflammation Research, Norwegian University of Science and Technology (NTNU), Trondheim, Norway

${ }^{11}$ Division of Radiology and Nuclear Medicine, Oslo University Hospital Rikshospitalet, Oslo, Norway

${ }^{12}$ Department of Radiology and Nuclear Medicine, St. Olavs Hospital, Trondheim University Hospital, Trondheim, Norway

${ }^{13}$ Department of Radiology, Oslo University Hospital Ullevaal, Oslo, Norway

${ }^{14}$ Department of Cardiology, Center for Clinical Heart Research, Oslo University Hospital Ullevaal, Oslo, Norway

${ }^{15}$ Department of Rheumatology, Dermatology and Infectious Disease, Oslo

University Hospital Rikshospitalet, Oslo, Norway

Acknowledgements The investigational medicinal products and an independent grant were kindly provided by Roche International.

Funding The ASSAIL-MI trial is an investigator-initiated study supported by unrestricted grants provided by the South-Eastern Norway Regional Health Authority. F.Hoffmann-La Roche Ltd, Grenacherstrasse 124, 4070 Basel, Switzerland has provided the investigational medicinal product used in this trial and has also provided an unrestricted sum for the implementation of the study. The funding sources have no role in the design of the study; neither will they participate in the implementation of the trial, in the analyses of the results, or in the decision to publish. The investigators take sole responsibility for the integrity of the data, the writing of the manuscript and the dissemination of the results.

Competing interests None declared.

Patient consent for publication Not required.

Ethics approval Regional Committee for Medical and Health Research Ethics of South-Eastern Norway and the Norwegian Medicines Agency.

Provenance and peer review Not commissioned; internally peer reviewed.

Data availability statement Data are available in a public, open access repository.

Open access This is an open access article distributed in accordance with the Creative Commons Attribution Non Commercial (CC BY-NC 4.0) license, which permits others to distribute, remix, adapt, build upon this work non-commercially, and license their derivative works on different terms, provided the original work is properly cited, appropriate credit is given, any changes made indicated, and the use is non-commercial. See: http://creativecommons.org/licenses/by-nc/4.0/.

\section{ORCID iD}

Anne Kristine Anstensrud http://orcid.org/0000-0003-1499-2402

\section{REFERENCES}

1 Timmis A, Townsend N, Gale C, et al. European Society of cardiology: cardiovascular disease statistics 2017. Eur Heart J 2018;39:508-79.

2 Benjamin EJ, Virani SS, Callaway CW, et al. Heart disease and stroke Statistics-2018 update: a report from the American heart association. Circulation 2018;137:e67-492.

3 Kleveland O, Kunszt G, Bratlie M, et al. Effect of a single dose of the interleukin- 6 receptor antagonist tocilizumab on inflammation and troponin T release in patients with non-ST-elevation myocardial infarction: a double-blind, randomized, placebo-controlled phase 2 trial. Eur Heart J 2016;37:2406-13.

4 Libby P, Pasterkamp G, Crea F, et al. Reassessing the mechanisms of acute coronary syndromes. Circ Res 2019;124:150-60.

5 Braunwald E, Kloner RA. Myocardial reperfusion: a double-edged sword? J Clin Invest 1985;76:1713-9.

6 Yellon DM, Hausenloy DJ. Myocardial reperfusion injury. $N$ Engl J Med 2007;357:1121-35.

7 Libby P. Molecular bases of the acute coronary syndromes. Circulation 1995;91:2844-50.

8 Hansson GK. Inflammation, atherosclerosis, and coronary artery disease. N Engl J Med 2005;352:1685-95.

9 Ridker PM, Everett BM, Thuren T, et al. Antiinflammatory therapy with canakinumab for atherosclerotic disease. N Engl J Med 2017;377:1119-31.

10 Morton AC, Rothman AMK, Greenwood JP, et al. The effect of interleukin-1 receptor antagonist therapy on markers of inflammation in non-ST elevation acute coronary syndromes: the MRC-ILA heart study. Eur Heart J 2015;36:377-84.

11 Van Tassell BW, Lipinski MJ, Appleton D, et al. Rationale and design of the Virginia Commonwealth University-Anakinra remodeling Trial-3 (VCU-ART3): a randomized, placebo-controlled, double-blinded, multicenter study. Clin Cardiol 2018;41:1004-8.

12 Granger CB, Mahaffey KW, Weaver WD, et al. Pexelizumab, an anti-C5 complement antibody, as adjunctive therapy to primary percutaneous coronary intervention in acute myocardial infarction: the complement inhibition in myocardial infarction treated with angioplasty (CoMMA) trial. Circulation 2003;108:1184-90.

13 Cung T-T, Morel O, Cayla G, et al. Cyclosporine before PCI in patients with acute myocardial infarction. $N$ Engl $\mathrm{J}$ Med 2015;373:1021-31.

14 Mahaffey KW, Granger CB, Nicolau JC, et al. Effect of pexelizumab, an anti-C5 complement antibody, as adjunctive therapy to fibrinolysis in acute myocardial infarction: the complement inhibition in myocardial infarction treated with thromboLYtics (COMPLY) trial. Circulation 2003;108:1176-83.

15 Deftereos S, Giannopoulos G, Angelidis C, et al. Anti-Inflammatory treatment with colchicine in acute myocardial infarction: a pilot study. Circulation 2015;132:1395-403.

16 Tardif J-C, Tanguay J-F, Wright SR, et al. Effects of the P-selectin antagonist inclacumab on myocardial damage after percutaneous coronary intervention for non-ST-segment elevation myocardial infarction: results of the SELECT-ACS trial. J Am Coll Cardiol 2013;61:2048-55.

17 O'Donoghue ML, Glaser R, Cavender MA, et al. Effect of Losmapimod on cardiovascular outcomes in patients hospitalized with acute myocardial infarction: a randomized clinical trial. JAMA 2016;315:1591-9.

18 Biasillo G, Leo M, Della Bona R, et al. Inflammatory biomarkers and coronary heart disease: from bench to bedside and back. Intern Emerg Med 2010;5:225-33.

19 Kinlay S, Schwartz GG, Olsson AG, et al. High-Dose atorvastatin enhances the decline in inflammatory markers in patients with acute coronary syndromes in the MIRACL study. Circulation 2003;108:1560-6.

20 Hou T, Tieu BC, Ray S, et al. Roles of IL-6-gp130 signaling in vascular inflammation. Curr Cardiol Rev 2008;4:179-92.

21 Huang $M$, Yang $D$, Xiang $M$, et al. Role of interleukin- 6 in regulation of immune responses to remodeling after myocardial infarction. Heart Fail Rev 2015;20:25-38.

22 Fontes JA, Rose NR, Čiháková D. The varying faces of IL-6: from cardiac protection to cardiac failure. Cytokine 2015;74:62-8.

23 Wollert KC, Drexler H. The role of interleukin- 6 in the failing heart. Heart Fail Rev 2001;6:95-103.

24 Kobara M, Noda K, Kitamura M, et al. Antibody against interleukin-6 receptor attenuates left ventricular remodelling after myocardial infarction in mice. Cardiovasc Res 2010;87:424-30.

25 Ritschel VN, Seljeflot I, Arnesen H, et al. Circulating levels of IL-6 receptor and gp130 and long-term clinical outcomes in ST-elevation myocardial infarction. J Am Heart Assoc 2016;5. doi:10.1161/ JAHA.115.003014 
26 Ridker PM, Libby P, MacFadyen JG, et al. Modulation of the interleukin- 6 signalling pathway and incidence rates of atherosclerotic events and all-cause mortality: analyses from the canakinumab anti-inflammatory thrombosis outcomes study (CANTOS). Eur Heart J 2018;39:3499-507.

27 Kleveland O, Ueland T, Kunszt G, et al. Interleukin-6 receptor inhibition with tocilizumab induces a selective and substantial increase in plasma IP-10 and MIP-1 $\beta$ in non-ST-elevation myocardial infarction. Int J Cardiol 2018;271:1-7.

28 Naruko T, Ueda M, Haze K, et al. Neutrophil infiltration of culprit lesions in acute coronary syndromes. Circulation 2002;106:2894-900.

29 Ueland T, Kleveland O, Michelsen AE, et al. Serum lipoprotein(a) is not modified by interleukin- 6 receptor antagonism or associated with inflammation in non-ST-elevation myocardial infarction. Int $J$ Cardiol 2019;274:348-50.

30 Eitel I, de Waha S, Wöhrle J, et al. Comprehensive prognosis assessment by CMR imaging after ST-segment elevation myocardial infarction. J Am Coll Cardiol 2014;64:1217-26.

31 Erlinge D, Götberg M, Lang I, et al. Rapid endovascular catheter core cooling combined with cold saline as an adjunct to percutaneous coronary intervention for the treatment of acute myocardial infarction. The CHILL-MI trial: a randomized controlled study of the use of central venous catheter core cooling combined with cold saline as an adjunct to percutaneous coronary intervention for the treatment of acute myocardial infarction. J Am Coll Cardiol 2014;63:1857-65.
32 Atar D, Arheden $\mathrm{H}$, Berdeaux A, et al. Effect of intravenous TRO40303 as an adjunct to primary percutaneous coronary intervention for acute ST-elevation myocardial infarction: MITOCARE study results. Eur Heart J 2015;36:112-9.

33 Ibanez B, Macaya C, Sánchez-Brunete V, et al. Effect of early metoprolol on infarct size in ST-segment-elevation myocardia infarction patients undergoing primary percutaneous coronary intervention: the effect of metoprolol in cardioprotection during an acute myocardial infarction (METOCARD-CNIC) trial. Circulation 2013;128:1495-503.

34 Eitel I, Stiermaier T, Rommel KP, et al. Cardioprotection by combined intrahospital remote ischaemic perconditioning and postconditioning in ST-elevation myocardial infarction: the randomized LIPSIA conditioning trial. Eur Heart J 2015;36:3049-57.

35 Regenfus M, Schlundt C, Krähner R, et al. Six-Year prognostic value of microvascular obstruction after reperfused ST-elevation myocardial infarction as assessed by contrast-enhanced cardiovascular magnetic resonance. Am J Cardiol 2015;116:1022-7.

36 Lunde K, Solheim S, Aakhus S, et al. Intracoronary injection of mononuclear bone marrow cells in acute myocardial infarction. $N$ Engl J Med 2006;355:1199-209.

37 Gibbons RJ, Valeti US, Araoz PA, et al. The quantification of infarct size. J Am Coll Cardiol 2004;44:1533-42.

38 Engblom H, Heiberg E, Erlinge D, et al. Sample size in clinical cardioprotection trials using myocardial salvage index, infarct size, or biochemical markers as endpoint. J Am Heart Assoc 2016;5:e002708. 